
complex precursor mixtures: influence of precursor volatility and molecular structure

S. H. Jathar et al.

Correspondence to: A. L. Robinson (alr@andrew.cmu.edu) 
Table S1: Composition information for California summer gasoline

\begin{tabular}{|c|c|c|c|c|c|}
\hline \multirow[b]{2}{*}{ Species } & \multirow[b]{2}{*}{$\begin{array}{c}\text { Mass } \\
\%\end{array}$} & \multirow[b]{2}{*}{ 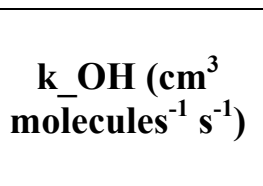 } & \multicolumn{3}{|c|}{ Lumping } \\
\hline & & & $\begin{array}{c}\text { CMAQ- } \\
\text { SAPRC } \\
\text { (speciated) }\end{array}$ & $\begin{array}{c}\text { CMAQ- } \\
\text { SAPRC (all) }\end{array}$ & $\begin{array}{c}\text { CMAQ-SAPRC } \\
\text { (alkane-resolved) }\end{array}$ \\
\hline C4 Linear Alkane & 0.3 & $2.36 \mathrm{E}-12$ & NONE & NONE & \multirow{8}{*}{$\begin{array}{l}\text { Lumped into } \\
n \text {-dodecane } \\
\text { equivalent } \\
\text { precursor as per } \\
\text { Table S7 }\end{array}$} \\
\hline C5 Linear Alkane & 10.9 & $3.80 \mathrm{E}-12$ & NONE & NONE & \\
\hline C6 Linear Alkane & 12.3 & $5.20 \mathrm{E}-12$ & NONE & NONE & \\
\hline C7 Linear Alkane & 9.4 & $6.76 \mathrm{E}-12$ & NONE & NONE & \\
\hline C8 Linear Alkane & 9.8 & $8.11 \mathrm{E}-12$ & ALK5 & ALK5 & \\
\hline C9 Linear Alkane & 3.1 & $9.70 \mathrm{E}-12$ & ALK5 & ALK5 & \\
\hline C10 Linear Alkane & 1.2 & $1.10 \mathrm{E}-11$ & ALK5 & ALK5 & \\
\hline C11+ Linear Alkane & 0.7 & $1.23 \mathrm{E}-11$ & ALK5 & ALK5 & \\
\hline C6 Aromatic & 0.6 & $1.22 \mathrm{E}-12$ & BENZ & BENZ & BENZ \\
\hline C7 Aromatic & 5.8 & $5.63 \mathrm{E}-12$ & ARO1 & ARO1 & ARO1 \\
\hline C8 Aromatic & 9.3 & $7.90 \mathrm{E}-12$ & ARO1 & ARO1 & ARO1 \\
\hline C9 Aromatic & 8.8 & $1.48 \mathrm{E}-11$ & $\mathrm{ARO} 2$ & $\mathrm{ARO} 2$ & ARO2 \\
\hline C10 Aromatic & 2.7 & $2.61 \mathrm{E}-11$ & $\mathrm{ARO} 2$ & ARO2 & ARO2 \\
\hline C11+ Aromatic & 1.0 & $4.35 \mathrm{E}-11$ & $\mathrm{ARO} 2$ & $\mathrm{ARO} 2$ & $\mathrm{ARO} 2$ \\
\hline Alkenes & 14.0 & $3.70 \mathrm{E}-11$ & NONE & NONE & NONE \\
\hline C5 Cyclo Alkane & 0.1 & $4.97 \mathrm{E}-12$ & NONE & NONE & \multirow{5}{*}{$\begin{array}{l}\text { Lumped into } \\
n \text {-dodecane } \\
\text { equivalent } \\
\text { precursor as per } \\
\text { Table S7 }\end{array}$} \\
\hline C6 Cyclo Alkane & 0.2 & $6.97 \mathrm{E}-12$ & ALK5 & ALK5 & \\
\hline C7 Cyclo Alkane & 0.1 & $1.24 \mathrm{E}-11$ & ALK5 & ALK5 & \\
\hline C8 Cyclo Alkane & 0.0 & $1.33 \mathrm{E}-11$ & ALK5 & ALK5 & \\
\hline C9 Cyclo Alkane & 0.0 & $1.60 \mathrm{E}-11$ & ALK5 & ALK5 & \\
\hline
\end{tabular}

Table S2: Composition information for Fischer-Tropsch (coal)

\begin{tabular}{|c|c|c|c|c|c|}
\hline \multirow[b]{2}{*}{ Species } & \multirow[b]{2}{*}{$\begin{array}{c}\text { Mass } \\
\%\end{array}$} & \multirow[b]{2}{*}{$\begin{array}{c}\text { k_OH }\left(\mathrm{cm}^{3}\right. \\
\left.\text { molecules }^{-1} \mathrm{~s}^{-1}\right)\end{array}$} & \multicolumn{3}{|c|}{ Lumping } \\
\hline & & & $\begin{array}{c}\text { CMAQ- } \\
\text { SAPRC } \\
\text { (speciated) }\end{array}$ & $\begin{array}{c}\text { CMAQ- } \\
\text { SAPRC } \\
\text { (all) }\end{array}$ & $\begin{array}{l}\text { CMAQ-SAPRC } \\
\text { (alkane-resolved) }\end{array}$ \\
\hline Linear Alkanes $\left(\sim \mathrm{C}_{10}\right)$ & 0.2 & $1.10 \mathrm{E}-11$ & ALK5 & ALK5 & \multirow{3}{*}{$\begin{array}{l}\text { Lumped into } \\
n \text {-dodecane } \\
\text { equivalent } \\
\text { precursor as per } \\
\text { Table S7 }\end{array}$} \\
\hline Branched Alkanes $\left(\sim \mathrm{C}_{10}\right)$ & 84.9 & $1.10 \mathrm{E}-11$ & $100 \%$ ALK5 & ALK5 & \\
\hline Cyclo Alkanes $\left(\sim \mathrm{C}_{10}\right)$ & 12.6 & $1.10 \mathrm{E}-11$ & $100 \%$ ALK5 & ALK5 & \\
\hline monoaromatics & 0.5 & $1.43 \mathrm{E}-11$ & ARO2 & ARO2 & ARO2 \\
\hline diaromatics & 0.1 & $2.30 \mathrm{E}-11$ & ARO2 & ARO2 & $\mathrm{ARO} 2$ \\
\hline
\end{tabular}


Table S3: Composition information for Fischer-Tropsch (natural gas)

\begin{tabular}{|c|c|c|c|c|c|}
\hline \multirow[b]{2}{*}{ Species } & \multirow[b]{2}{*}{ Mass \% } & \multirow[b]{2}{*}{$\begin{array}{c}\mathrm{k} \_\mathrm{OH}\left(\mathrm{cm}^{3}\right. \\
\left.\text { molecules }^{-1} \mathrm{~s}^{-1}\right)\end{array}$} & \multicolumn{3}{|c|}{ Lumping } \\
\hline & & & $\begin{array}{c}\text { CMAQ- } \\
\text { SAPRC } \\
\text { (speciated) } \\
\end{array}$ & $\begin{array}{c}\text { CMAQ- } \\
\text { SAPRC } \\
\text { (all) }\end{array}$ & $\begin{array}{l}\text { CMAQ-SAPRC } \\
\text { (alkane-resolved) }\end{array}$ \\
\hline C7 Linear Alkane & 0.0 & $6.76 \mathrm{E}-12$ & NONE & NONE & \multirow{14}{*}{$\begin{array}{l}\text { Lumped into } \\
\text { n-dodecane } \\
\text { equivalent precursor } \\
\text { as per Table S7 }\end{array}$} \\
\hline C8 Linear Alkane & 1.6 & $8.11 \mathrm{E}-12$ & ALK5 & ALK5 & \\
\hline C9 Linear Alkane & 22.4 & $9.70 \mathrm{E}-12$ & ALK5 & ALK5 & \\
\hline C10 Linear Alkane & 25.1 & $1.10 \mathrm{E}-11$ & ALK5 & ALK5 & \\
\hline C11 Linear Alkane & 3.8 & $1.23 \mathrm{E}-11$ & ALK5 & ALK5 & \\
\hline C12 Linear Alkane & 0.3 & $1.32 \mathrm{E}-11$ & ALK5 & ALK5 & \\
\hline C13 Linear Alkane & 0.0 & $1.51 \mathrm{E}-11$ & NONE & ALK5 & \\
\hline C14 Linear Alkane & 0.0 & $1.79 \mathrm{E}-11$ & NONE & ALK5 & \\
\hline C15 Linear Alkane & 0.0 & $2.07 \mathrm{E}-11$ & NONE & ALK5 & \\
\hline C16 Linear Alkane & 0.0 & $2.32 \mathrm{E}-11$ & NONE & ALK5 & \\
\hline C17 Linear Alkane & 0.0 & $2.85 \mathrm{E}-11$ & NONE & ALK5 & \\
\hline C18 Linear Alkane & 0.0 & $3.51 \mathrm{E}-11$ & NONE & ALK5 & \\
\hline C19 Linear Alkane & 0.0 & $4.32 \mathrm{E}-11$ & NONE & ALK5 & \\
\hline $\begin{array}{l}\text { Branched Alkanes } \\
\left(\sim \mathrm{C}_{10}\right)\end{array}$ & 46.8 & $1.10 \mathrm{E}-11$ & $100 \%$ ALK5 & ALK5 & \\
\hline
\end{tabular}

Table S4: Composition information for Jet Propellent-8

\begin{tabular}{|c|c|c|c|c|c|}
\hline \multirow[b]{2}{*}{ Species } & \multirow[b]{2}{*}{ Mass \% } & \multirow{2}{*}{$\begin{array}{c}\text { k_OH }\left(\mathrm{cm}^{3}\right. \\
\left.\text { molecules }^{-1} \mathrm{~s}^{-1}\right)\end{array}$} & \multicolumn{3}{|c|}{ Lumping } \\
\hline & & & $\begin{array}{c}\text { CMAQ-SAPRC } \\
\text { (speciated) }\end{array}$ & $\begin{array}{c}\text { CMAQ- } \\
\text { SAPRC (all) } \\
\end{array}$ & $\begin{array}{c}\text { CMAQ-SAPRC } \\
\text { (alkane-resolved) }\end{array}$ \\
\hline C7 Linear Alkane & 0.1 & $6.76 \mathrm{E}-12$ & NONE & NONE & \multirow{15}{*}{$\begin{array}{l}\text { Lumped into } \\
\text { n-dodecane } \\
\text { equivalent } \\
\text { precursor as per } \\
\text { Table S7 }\end{array}$} \\
\hline C8 Linear Alkane & 0.3 & $8.11 \mathrm{E}-12$ & ALK5 & ALK5 & \\
\hline C9 Linear Alkane & 1.2 & $9.70 \mathrm{E}-12$ & ALK5 & ALK5 & \\
\hline C10 Linear Alkane & 3.5 & $1.10 \mathrm{E}-11$ & ALK5 & ALK5 & \\
\hline C11 Linear Alkane & 4.2 & $1.23 \mathrm{E}-11$ & ALK5 & ALK5 & \\
\hline C12 Linear Alkane & 3.7 & $1.32 \mathrm{E}-11$ & ALK5 & ALK5 & \\
\hline C13 Linear Alkane & 2.8 & $1.51 \mathrm{E}-11$ & NONE & ALK5 & \\
\hline C14 Linear Alkane & 1.8 & $1.79 \mathrm{E}-11$ & NONE & ALK5 & \\
\hline C15 Linear Alkane & 0.9 & $2.07 \mathrm{E}-11$ & NONE & ALK5 & \\
\hline C16 Linear Alkane & 0.3 & $2.32 \mathrm{E}-11$ & NONE & ALK5 & \\
\hline C17 Linear Alkane & 0.1 & $2.85 \mathrm{E}-11$ & NONE & ALK5 & \\
\hline C18 Linear Alkane & 0.0 & $3.51 \mathrm{E}-11$ & NONE & ALK5 & \\
\hline C19 Linear Alkane & 0.0 & 4.32E-11 & NONE & ALK5 & \\
\hline $\begin{array}{l}\text { Branched Alkanes } \\
\left(\sim \mathrm{C}_{11}\right)\end{array}$ & 23.1 & $1.43 \mathrm{E}-11$ & $69 \%$ ALK5 & ALK5 & \\
\hline Cyclo Alkanes $\left(\sim \mathrm{C}_{11}\right)$ & 34.2 & $2.30 \mathrm{E}-11$ & $69 \%$ ALK5 & ALK5 & \\
\hline monoaromatics & 15.4 & $1.23 \mathrm{E}-11$ & $\mathrm{ARO} 2$ & $\mathrm{ARO} 2$ & $\mathrm{ARO} 2$ \\
\hline diaromatics & 1.7 & $1.23 \mathrm{E}-11$ & $\mathrm{ARO} 2$ & $\mathrm{ARO} 2$ & $\mathrm{ARO} 2$ \\
\hline
\end{tabular}


Table S5: Composition information for diesels

\begin{tabular}{|c|c|c|c|c|c|c|c|c|c|c|c|}
\hline \multirow[b]{2}{*}{ Species } & \multicolumn{7}{|c|}{ Mass \% } & \multirow[b]{2}{*}{$\underset{\left.\text { molecules }^{-1} \mathbf{s}^{-1}\right)}{\mathbf{k}_{-} \mathrm{OH}\left(\mathrm{cm}^{3}\right.}$} & \multicolumn{3}{|c|}{ Lumping } \\
\hline & (1) & (2) & (3) & (5) & (7) & (8) & (9) & & $\begin{array}{l}\text { CMAQ- } \\
\text { SAPRC } \\
\text { (speciated) }\end{array}$ & $\begin{array}{c}\text { CMAQ- } \\
\text { SAPRC (all) }\end{array}$ & $\begin{array}{c}\text { CMAQ-SAPRC } \\
\text { (alkane-resolved) }\end{array}$ \\
\hline C3 Linear Alkane & 0.0 & 0.0 & 0.0 & 0.0 & 0.0 & 0.0 & 0.0 & $1.09 \mathrm{E}-12$ & NONE & NONE & Lumped into \\
\hline C4 Linear Alkane & 0.0 & 0.0 & 0.0 & 0.0 & 0.0 & 0.0 & 0.0 & $2.36 \mathrm{E}-12$ & NONE & NONE & n-dodecane \\
\hline C5 Linear Alkane & 0.0 & 0.0 & 0.0 & 0.0 & 0.0 & 0.0 & 0.0 & $3.80 \mathrm{E}-12$ & NONE & NONE & equivalent \\
\hline C6 Linear Alkane & 0.0 & 0.0 & 0.0 & 0.0 & 0.0 & 0.0 & 0.0 & $5.20 \mathrm{E}-12$ & NONE & NONE & precursor as per \\
\hline C7 Linear Alkane & 0.0 & 0.1 & 0.0 & 0.0 & 0.0 & 0.0 & 0.0 & $6.76 \mathrm{E}-12$ & NONE & NONE & Table S7 \\
\hline C8 Linear Alkane & 0.0 & 0.0 & 0.2 & 0.1 & 0.1 & 0.0 & 0.1 & $8.11 \mathrm{E}-12$ & ALK5 & ALK5 & \\
\hline C9 Linear Alkane & 0.3 & 0.2 & 0.6 & 0.7 & 0.5 & 0.0 & 0.4 & $9.70 \mathrm{E}-12$ & ALK5 & ALK5 & \\
\hline C10 Linear Alkane & 0.4 & 0.2 & 1.7 & 2.9 & 1.5 & 0.1 & 0.8 & $1.10 \mathrm{E}-11$ & ALK5 & ALK5 & \\
\hline C11 Linear Alkane & 0.4 & 0.2 & 1.4 & 2.9 & 2.0 & 0.1 & 1.1 & $1.23 \mathrm{E}-11$ & ALK5 & ALK5 & \\
\hline C12 Linear Alkane & 0.2 & 0.1 & 2.0 & 3.5 & 1.7 & 0.1 & 0.8 & $1.32 \mathrm{E}-11$ & ALK5 & ALK5 & \\
\hline C13 Linear Alkane & 0.0 & 0.0 & 1.3 & 2.4 & 1.2 & 0.4 & 0.9 & $1.51 \mathrm{E}-11$ & NONE & ALK5 & \\
\hline C14 Linear Alkane & 0.2 & 0.0 & 1.3 & 12.0 & 18.2 & 16.4 & 1.2 & $1.79 \mathrm{E}-11$ & NONE & ALK5 & \\
\hline C15 Linear Alkane & 0.5 & 0.0 & 1.4 & 7.0 & 10.1 & 8.5 & 1.7 & $2.07 \mathrm{E}-11$ & NONE & ALK5 & \\
\hline C16 Linear Alkane & 0.6 & 0.0 & 1.0 & 1.0 & 1.5 & 1.1 & 1.0 & $2.32 \mathrm{E}-11$ & NONE & ALK5 & \\
\hline C17 Linear Alkane & 0.5 & 0.0 & 0.6 & 0.0 & 0.1 & 0.3 & 1.0 & $2.85 \mathrm{E}-11$ & NONE & ALK5 & \\
\hline C18 Linear Alkane & 0.8 & 0.0 & 0.3 & 0.0 & 0.0 & 0.0 & 0.4 & $3.51 \mathrm{E}-11$ & NONE & ALK5 & \\
\hline C19 Linear Alkane & 0.6 & 0.0 & 0.0 & 0.0 & 0.0 & 0.0 & 0.2 & $4.32 \mathrm{E}-11$ & NONE & ALK5 & \\
\hline C20 Linear Alkane & 0.3 & 0.0 & 0.0 & 0.0 & 0.0 & 0.0 & 0.0 & $4.32 \mathrm{E}-11$ & NONE & ALK5 & \\
\hline C21 Linear Alkane & 0.0 & 0.0 & 0.0 & 0.0 & 0.0 & 0.0 & 0.0 & $4.32 \mathrm{E}-11$ & NONE & ALK5 & \\
\hline C3 Branched Alkane & 0.0 & 0.0 & 0.0 & 0.0 & 0.0 & 0.0 & 0.0 & $1.39 \mathrm{E}-12$ & NONE & NONE & \\
\hline C4 Branched Alkane & 0.0 & 0.0 & 0.0 & 0.0 & 0.0 & 0.0 & 0.0 & $2.63 \mathrm{E}-12$ & NONE & NONE & \\
\hline C5 Branched Alkane & 0.0 & 0.0 & 0.0 & 0.0 & 0.0 & 0.1 & 0.0 & $3.95 \mathrm{E}-12$ & NONE & NONE & \\
\hline C6 Branched Alkane & 0.0 & 0.0 & 0.0 & 0.0 & 0.0 & 0.0 & 0.0 & 5.34E-12 & NONE & NONE & \\
\hline C7 Branched Alkane & 0.0 & 0.0 & 0.0 & 0.1 & 0.0 & 0.0 & 0.0 & $6.81 \mathrm{E}-12$ & NONE & NONE & \\
\hline C8 Branched Alkane & 0.1 & 0.1 & 0.4 & 0.2 & 0.1 & 0.1 & 0.2 & $8.35 \mathrm{E}-12$ & ALK5 & ALK5 & \\
\hline C9 Branched Alkane & 0.5 & 0.1 & 0.7 & 0.8 & 0.6 & 0.0 & 0.0 & $9.96 \mathrm{E}-12$ & ALK5 & ALK5 & \\
\hline C10 Branched Alkane & 2.0 & 1.0 & 2.9 & 3.3 & 1.6 & 0.0 & 1.1 & $1.17 \mathrm{E}-11$ & ALK5 & ALK5 & \\
\hline C11 Branched Alkane & 4.7 & 4.5 & 3.2 & 2.0 & 0.4 & 0.0 & 0.2 & $1.34 \mathrm{E}-11$ & ALK5 & ALK5 & \\
\hline C12 Branched Alkane & 0.5 & 1.1 & 0.9 & 2.3 & 0.8 & 0.0 & 0.1 & $1.53 \mathrm{E}-11$ & ALK5 & ALK5 & \\
\hline
\end{tabular}




\begin{tabular}{|c|c|c|c|c|c|c|c|c|c|c|c|}
\hline \multirow[b]{2}{*}{ Species } & \multicolumn{7}{|c|}{ Mass \% } & \multirow[b]{2}{*}{$\begin{array}{c}\text { k_OH }\left(\mathrm{cm}^{3}\right. \\
\left.\text { molecules }^{-1} \mathbf{s}^{-1}\right)\end{array}$} & \multicolumn{3}{|c|}{ Lumping } \\
\hline & (1) & (2) & (3) & (5) & $(7)$ & (8) & (9) & & $\begin{array}{l}\text { CMAQ- } \\
\text { SAPRC } \\
\text { (speciated) }\end{array}$ & $\begin{array}{c}\text { CMAQ- } \\
\text { SAPRC (all) }\end{array}$ & $\begin{array}{l}\text { CMAQ-SAPRC } \\
\text { (alkane-resolved) }\end{array}$ \\
\hline C13 Branched Alkane & 3.0 & 7.8 & 3.1 & 3.1 & 1.5 & 0.0 & 1.0 & $1.72 \mathrm{E}-11$ & NONE & ALK5 & \\
\hline C14 Branched Alkane & 4.1 & 5.5 & 1.9 & 2.3 & 1.0 & 1.8 & 3.0 & $1.92 \mathrm{E}-11$ & NONE & ALK5 & \\
\hline C15 Branched Alkane & 3.9 & 1.1 & 2.8 & 1.0 & 0.3 & 1.5 & 3.3 & $2.12 \mathrm{E}-11$ & NONE & ALK5 & \\
\hline C16 Branched Alkane & 3.6 & 0.6 & 1.1 & 0.5 & 0.2 & 1.1 & 3.3 & $2.33 \mathrm{E}-11$ & NONE & ALK5 & \\
\hline C17 Branched Alkane & 2.5 & 0.2 & 0.3 & 0.0 & 0.0 & 1.1 & 2.6 & $2.56 \mathrm{E}-11$ & NONE & ALK5 & \\
\hline C18 Branched Alkane & 1.5 & 0.1 & 0.0 & 0.0 & 0.0 & 0.0 & 1.2 & $2.78 \mathrm{E}-11$ & NONE & ALK5 & \\
\hline C19 Branched Alkane & 1.6 & 0.1 & 0.0 & 0.0 & 0.0 & 0.0 & 0.2 & $3.02 \mathrm{E}-11$ & NONE & ALK5 & \\
\hline C20 Branched Alkane & 0.7 & 0.0 & 0.0 & 0.0 & 0.0 & 0.0 & 0.0 & $3.26 \mathrm{E}-11$ & NONE & ALK5 & \\
\hline C21 Branched Alkane & 0.2 & 0.0 & 0.0 & 0.0 & 0.0 & 0.0 & 0.0 & $3.51 \mathrm{E}-11$ & NONE & ALK5 & \\
\hline C3 Cyclo Alkane & 0.0 & 0.0 & 0.0 & 0.0 & 0.0 & 0.0 & 0.0 & $1.39 \mathrm{E}-12$ & NONE & NONE & \\
\hline C4 Cyclo Alkane & 0.0 & 0.0 & 0.0 & 0.0 & 0.0 & 0.0 & 0.0 & $2.63 \mathrm{E}-12$ & NONE & NONE & \\
\hline C5 Cyclo Alkane & 0.0 & 0.0 & 0.0 & 0.0 & 0.0 & 0.0 & 0.0 & $3.95 \mathrm{E}-12$ & NONE & NONE & \\
\hline C6 Cyclo Alkane & 0.0 & 0.0 & 0.0 & 0.0 & 0.0 & 0.0 & 0.0 & 5.34E-12 & NONE & NONE & \\
\hline C7 Cyclo Alkane & 0.0 & 0.0 & 0.1 & 0.0 & 0.0 & 0.0 & 0.0 & $6.81 \mathrm{E}-12$ & NONE & NONE & \\
\hline C8 Cyclo Alkane & 0.3 & 0.0 & 0.4 & 0.4 & 0.2 & 0.0 & 0.3 & $8.35 \mathrm{E}-12$ & ALK5 & ALK5 & \\
\hline C9 Cyclo Alkane & 0.6 & 0.1 & 0.8 & 1.0 & 0.8 & 0.0 & 0.9 & $9.96 \mathrm{E}-12$ & ALK5 & ALK5 & \\
\hline C10 Cyclo Alkane & 1.0 & 0.1 & 1.7 & 2.9 & 1.4 & 0.0 & 0.7 & $1.17 \mathrm{E}-11$ & ALK5 & ALK5 & \\
\hline C11 Cyclo Alkane & 1.4 & 0.7 & 2.1 & 3.3 & 1.1 & 0.0 & 0.7 & $1.34 \mathrm{E}-11$ & ALK5 & ALK5 & \\
\hline C12 Cyclo Alkane & 1.1 & 2.4 & 4.3 & 5.8 & 2.8 & 0.0 & 2.3 & $1.53 \mathrm{E}-11$ & ALK5 & ALK5 & \\
\hline C13 Cyclo Alkane & 1.8 & 2.1 & 4.0 & 4.6 & 2.5 & 1.0 & 5.9 & $1.72 \mathrm{E}-11$ & NONE & ALK5 & \\
\hline C14 Cyclo Alkane & 2.3 & 1.5 & 2.4 & 1.7 & 1.4 & 1.0 & 3.2 & $1.92 \mathrm{E}-11$ & NONE & ALK5 & \\
\hline C15 Cyclo Alkane & 2.9 & 0.9 & 1.4 & 1.6 & 0.6 & 3.3 & 7.9 & $2.12 \mathrm{E}-11$ & NONE & ALK5 & \\
\hline C16 Cyclo Alkane & 2.3 & 0.7 & 0.5 & 2.5 & 0.2 & 2.3 & 4.5 & $2.33 \mathrm{E}-11$ & NONE & ALK5 & \\
\hline C17 Cyclo Alkane & 1.9 & 0.7 & 0.1 & 2.0 & 0.5 & 4.1 & 7.2 & $2.56 \mathrm{E}-11$ & NONE & ALK5 & \\
\hline C18 Cyclo Alkane & 1.5 & 1.0 & 0.0 & 0.9 & 0.0 & 1.0 & 2.3 & $2.78 \mathrm{E}-11$ & NONE & ALK5 & \\
\hline C19 Cyclo Alkane & 1.4 & 1.3 & 0.0 & 0.5 & 0.5 & 6.1 & 1.9 & $3.02 \mathrm{E}-11$ & NONE & ALK5 & \\
\hline C20 Cyclo Alkane & 1.0 & 1.2 & 0.0 & 0.2 & 0.0 & 1.5 & 1.1 & $3.26 \mathrm{E}-11$ & NONE & ALK5 & \\
\hline C21 Cyclo Alkane & 0.7 & 2.5 & 0.0 & 0.0 & 0.0 & 2.0 & 0.5 & $3.51 \mathrm{E}-11$ & NONE & ALK5 & \\
\hline C3 Aromatic & 0.0 & 0.0 & 0.0 & 0.0 & 0.0 & 0.0 & 0.0 & $0.00 \mathrm{E}+00$ & NONE & NONE & NONE \\
\hline C4 Aromatic & 0.0 & 0.0 & 0.0 & 0.0 & 0.0 & 0.0 & 0.0 & $0.00 \mathrm{E}+00$ & NONE & NONE & NONE \\
\hline
\end{tabular}




\begin{tabular}{|c|c|c|c|c|c|c|c|c|c|c|c|}
\hline \multirow[b]{2}{*}{ Species } & \multicolumn{7}{|c|}{ Mass \% } & \multirow[b]{2}{*}{$\begin{array}{c}\mathbf{k}_{\left.\text {molecules }^{-1} \mathbf{s}^{-1}\right)} \\
\text { OH }\left(\mathrm{cm}^{3}\right.\end{array}$} & \multicolumn{3}{|c|}{ Lumping } \\
\hline & (1) & (2) & (3) & (5) & (7) & (8) & (9) & & $\begin{array}{l}\text { CMAQ- } \\
\text { SAPRC } \\
\text { (speciated) }\end{array}$ & $\begin{array}{c}\text { CMAQ- } \\
\text { SAPRC (all) }\end{array}$ & $\begin{array}{c}\text { CMAQ-SAPRC } \\
\text { (alkane-resolved) }\end{array}$ \\
\hline C5 Aromatic & 0.0 & 0.0 & 0.0 & 0.0 & 0.0 & 0.0 & 0.0 & $0.00 \mathrm{E}+00$ & NONE & NONE & NONE \\
\hline C6 Aromatic & 0.1 & 0.0 & 0.0 & 0.0 & 0.0 & 0.0 & 0.0 & $1.22 \mathrm{E}-12$ & BENZ & BENZ & BENZ \\
\hline C7 Aromatic & 0.1 & 0.1 & 0.1 & 0.1 & 0.0 & 0.0 & 0.2 & $5.63 \mathrm{E}-12$ & ARO1 & ARO1 & ARO1 \\
\hline C8 Aromatic & 5.0 & 7.1 & 5.4 & 1.8 & 0.5 & 0.1 & 3.1 & $7.90 \mathrm{E}-12$ & ARO1 & $\mathrm{ARO} 2$ & $\mathrm{ARO} 2$ \\
\hline C9 Aromatic & 27.0 & 34.5 & 26.8 & 11.7 & 19.2 & 2.9 & 15.1 & $1.48 \mathrm{E}-11$ & ARO2 & $\mathrm{ARO} 2$ & ARO2 \\
\hline C10 Aromatic & 4.4 & 5.6 & 0.8 & 0.7 & 0.9 & 0.0 & 0.3 & $2.61 \mathrm{E}-11$ & ARO2 & ARO2 & ARO2 \\
\hline C11 Aromatic & 0.8 & 2.9 & 2.6 & 2.6 & 2.5 & 0.0 & 1.0 & $4.35 \mathrm{E}-11$ & ARO2 & ARO2 & ARO2 \\
\hline C12 Aromatic & 0.3 & 0.4 & 1.5 & 1.6 & 1.3 & 1.4 & 0.6 & $6.94 \mathrm{E}-11$ & $\mathrm{ARO} 2$ & $\mathrm{ARO} 2$ & $\mathrm{ARO} 2$ \\
\hline C13 Aromatic & 0.3 & 0.2 & 1.0 & 0.9 & 0.5 & 0.2 & 0.5 & $1.07 \mathrm{E}-10$ & NONE & ARO2 & ARO2 \\
\hline C14 Aromatic & 0.3 & 0.2 & 1.0 & 0.4 & 2.9 & 6.1 & 0.8 & $1.59 \mathrm{E}-10$ & NONE & $\mathrm{ARO} 2$ & ARO2 \\
\hline C15 Aromatic & 0.2 & 0.1 & 0.5 & 0.0 & 0.1 & 0.0 & 0.5 & $2.29 \mathrm{E}-10$ & NONE & $\mathrm{ARO} 2$ & ARO2 \\
\hline C16 Aromatic & 0.2 & 0.1 & 0.3 & 0.0 & 0.0 & 0.1 & 0.4 & $3.24 \mathrm{E}-10$ & NONE & $\mathrm{ARO} 2$ & $\mathrm{ARO} 2$ \\
\hline C17 Aromatic & 0.2 & 0.1 & 0.1 & 0.1 & 0.0 & 0.1 & 0.4 & $4.49 \mathrm{E}-10$ & NONE & $\mathrm{ARO} 2$ & $\mathrm{ARO} 2$ \\
\hline C18 Aromatic & 0.1 & 0.1 & 0.0 & 0.1 & 0.0 & 0.1 & 0.4 & $6.10 \mathrm{E}-10$ & NONE & $\mathrm{ARO} 2$ & $\mathrm{ARO} 2$ \\
\hline C19 Aromatic & 0.1 & 0.1 & 0.0 & 0.0 & 0.0 & 0.2 & 0.3 & $8.15 \mathrm{E}-10$ & NONE & $\mathrm{ARO} 2$ & $\mathrm{ARO} 2$ \\
\hline C20 Aromatic & 0.1 & 0.2 & 0.0 & 0.0 & 0.0 & 0.3 & 0.2 & $1.07 \mathrm{E}-09$ & NONE & $\mathrm{ARO} 2$ & ARO2 \\
\hline C21 Aromatic & 0.0 & 0.2 & 0.0 & 0.0 & 0.0 & 0.3 & 0.1 & 1.39E-09 & NONE & $\mathrm{ARO} 2$ & $\mathrm{ARO} 2$ \\
\hline benzocycloalkanes & 3.9 & 3.3 & 8.1 & 3.6 & 4.9 & 6.3 & 6.4 & $1.10 \mathrm{E}-10$ & ARO2 & ARO2 & ARO2 \\
\hline diaromatics & 1.5 & 3.6 & 4.4 & 0.9 & 11.9 & 26.5 & 4.4 & $2.30 \mathrm{E}-11$ & ARO2 & $\mathrm{ARO} 2$ & ARO2 \\
\hline triaromatics & 0.0 & 0.0 & 0.0 & 0.0 & 0.0 & 0.1 & 0.0 & $1.10 \mathrm{E}-10$ & NONE & $\mathrm{ARO} 2$ & ARO2 \\
\hline tetraaromatics & 0.0 & 0.0 & 0.0 & 0.0 & 0.0 & 0.0 & 0.0 & $1.10 \mathrm{E}-10$ & NONE & $\mathrm{ARO} 2$ & $\mathrm{ARO} 2$ \\
\hline alkenes & 1.8 & 2.5 & 0.1 & 0.2 & 0.1 & 0.0 & 0.1 & $5.00 \mathrm{E}-11$ & NONE & NONE & NONE \\
\hline
\end{tabular}


Table S6: SOA mass yields for CMAQ-SAPRC lumped species

\begin{tabular}{|l|c|c|c|c|}
\hline Species & $\alpha_{1}$ & $\mathrm{C}_{1}^{*}$ & $\alpha_{2}$ & $\mathrm{C}^{*}$ \\
\hline ALK5 & 0.0718 & 0.02 & - & - \\
\hline BENZ & 0.0942 & 0.302 & 1.162 & 111.11 \\
\hline ARO1 & 0.0758 & 2.326 & 0.1477 & 21.277 \\
\hline ARO2 & 0.0386 & 1.314 & 0.1119 & 34.483 \\
\hline$n$-dodecane & 0.03 & 0.147 & 0.22 & 51.9 \\
\hline
\end{tabular}

Table S7: SOA yields of linear, branched and cyclic alkanes relative to $n$-dodecane that can be used to determine an $n$-dodecane equivalent mass (Reproduced from Pye and Pouliot (2012)).

\begin{tabular}{|l|c|c|c|}
\hline Carbon number & Linear & Branched & Cyclic \\
\hline 6 & 0 & 0 & 0.11 \\
\hline 7 & 0 & 0 & 0.53 \\
\hline 8 & 0.1 & 0.05 & 0.96 \\
\hline 9 & 0.28 & 0.14 & 1.4 \\
\hline 10 & 0.67 & 0.34 & 1.8 \\
\hline 11 & 0.89 & 0.45 & 2.1 \\
\hline 12 & 1 & 0.5 & 2.3 \\
\hline 13 & 2.6 & 1.3 & 3.7 \\
\hline 14 & 2.9 & 1.5 & 5.2 \\
\hline 15 & 4.4 & 2.2 & 6.6 \\
\hline 16 & 5 & 2.5 & 6.6 \\
\hline $17-21$ & 5.6 & 2.8 & 6.6 \\
\hline
\end{tabular}



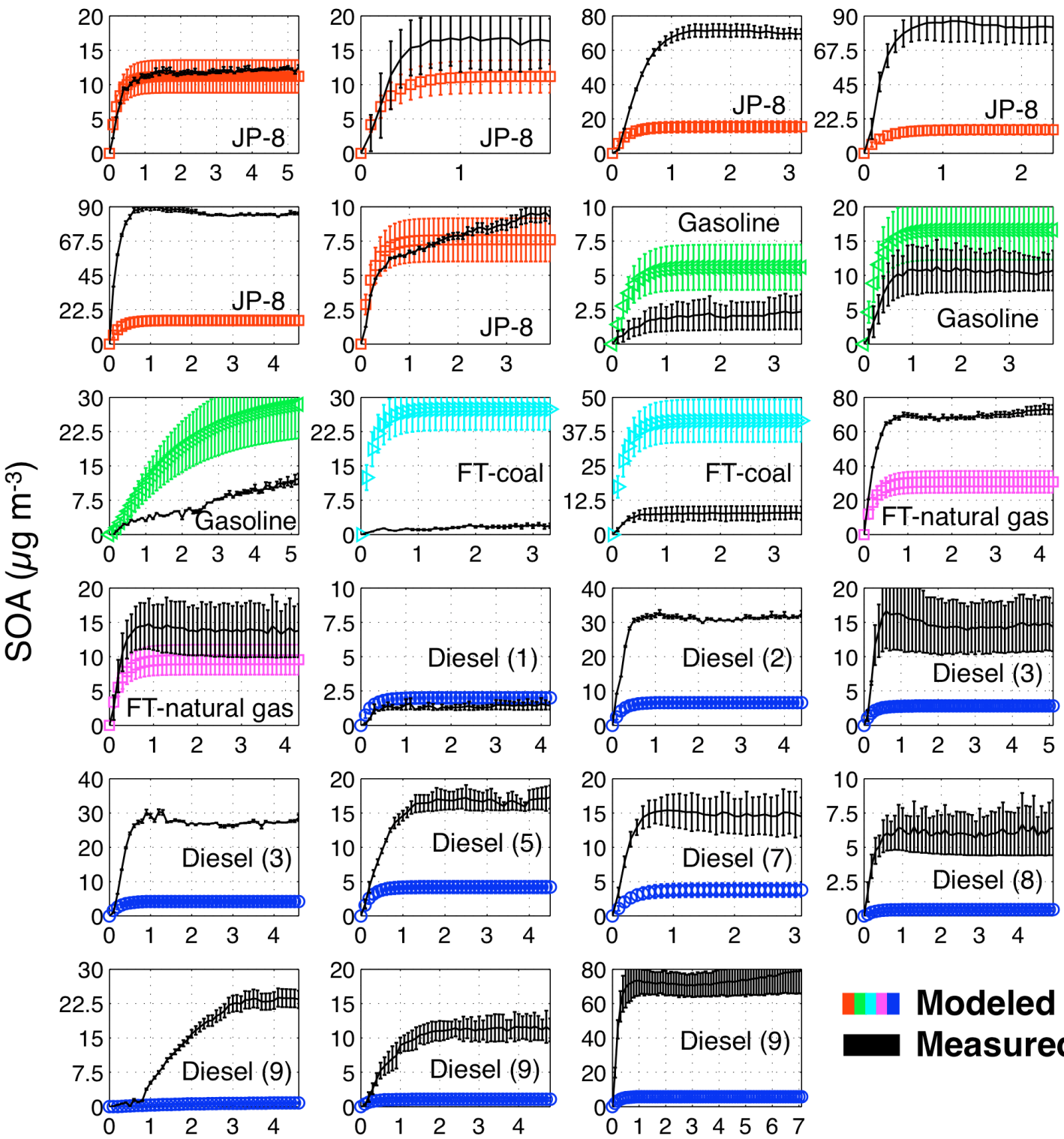

\section{Modeled Measured}

Time (hrs)

Figure S1: CMAQ-SAPRC (speciated) model predictions of SOA compared against measurements as a function of time for 23 experiments from Jathar et al. (2013). 

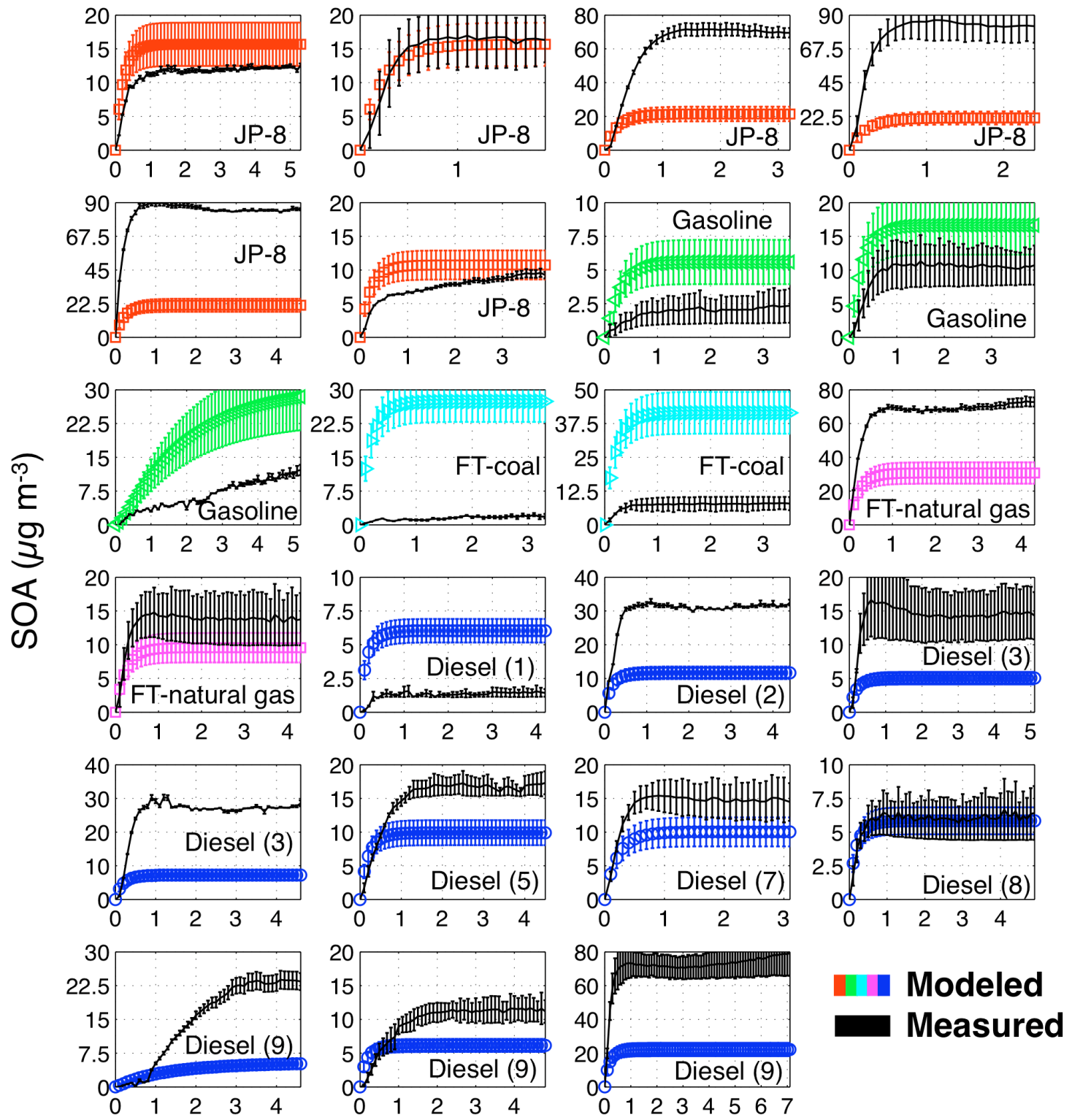

Time (hrs)

Figure S2: CMAQ-SAPRC (all) model predictions of SOA compared against measurements as a function of time for 23 experiments from Jathar et al. (2013). 

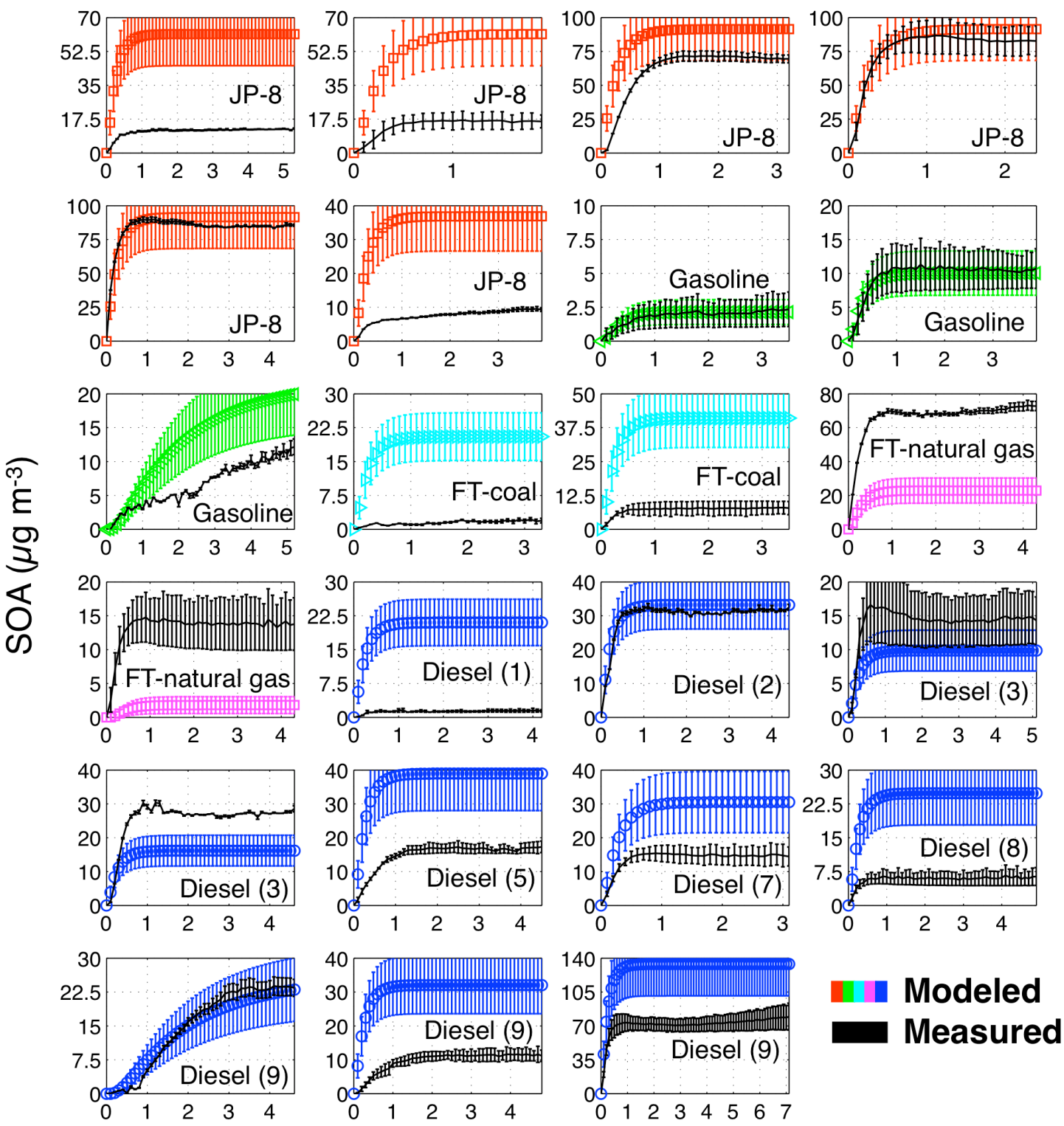

Time (hrs)

Figure S3: CMAQ-SAPRC (alkane-resolved) model predictions of SOA compared against measurements as a function of time for 23 experiments from Jathar et al. (2013). 

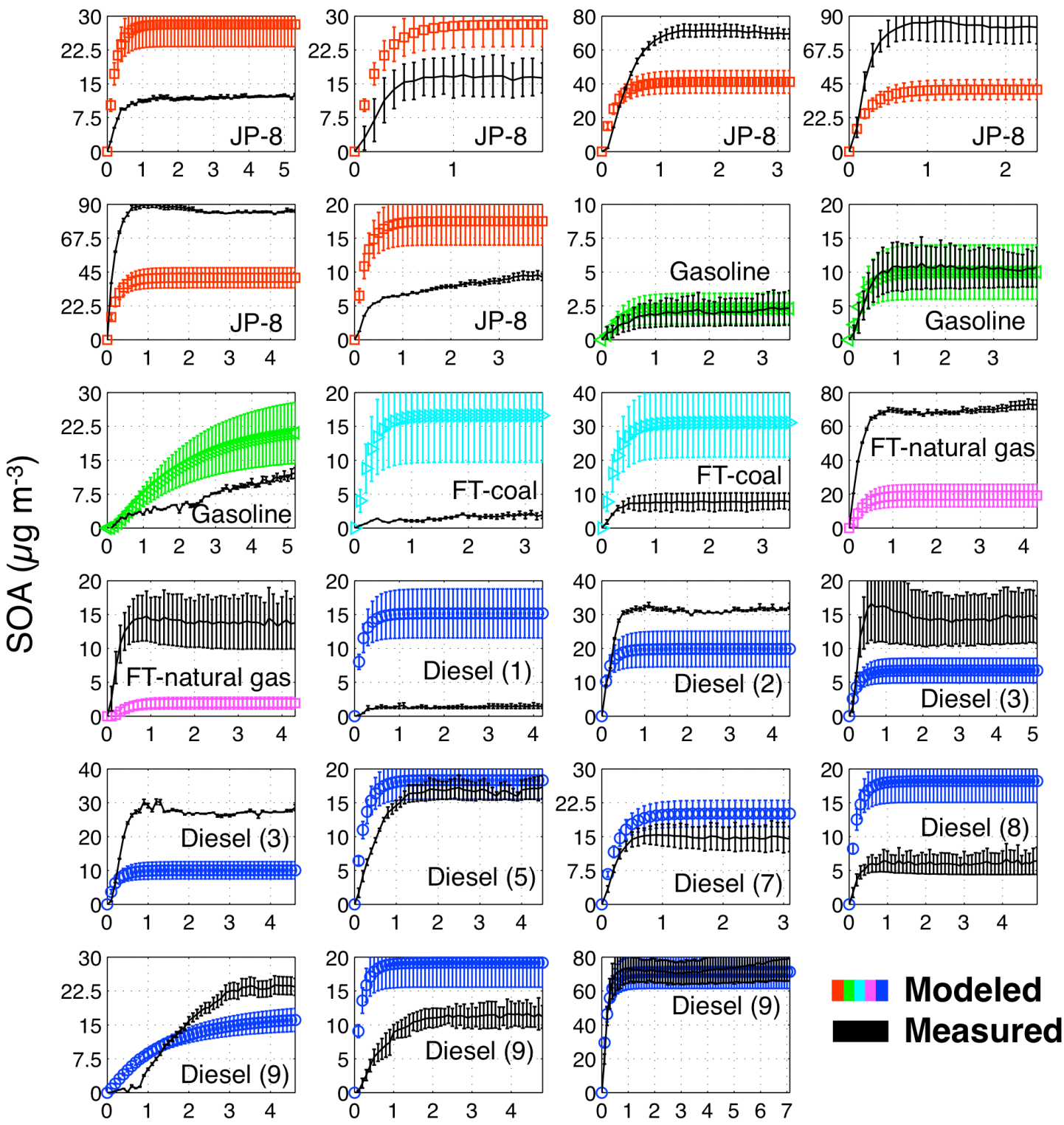

Time (hrs)

Figure S4: Volatility-Dependent model predictions of SOA compared against measurements as a function of time for 23 experiments from Jathar et al. (2013).

\section{References}

Jathar, S. H., Miracolo, M. A., Tkacik, D. S., Donahue, N. M., Adams, P. J., and Robinson, A. L.: Secondary Organic Aerosol Formation from Photo-Oxidation of Unburned Fuel:

Experimental Results and Implications for Aerosol Formation from Combustion Emissions, Environmental Science \& Technology, 47, 12886-12893, 10.1021/es403445q, 2013. 
Pye, H. O., and Pouliot, G. A.: Modeling the role of alkanes, polycyclic aromatic hydrocarbons, and their oligomers in secondary organic aerosol formation, Environmental Science \& Technology, 46, 6041-6047, 2012. 